\title{
Does Consciousness require a cerebral cortex?
}

\author{
Michel Dojat ${ }^{1}$, Manik Bhattacharjee ${ }^{1}$, Christian Graff ${ }^{2}$ \\ ${ }^{1}$ University Grenoble Alpes, Inserm U1216, Grenoble Institute of Neurosciences, Grenoble, Fr \\ ${ }^{2}$ Laboratory of Psychology and Neurocognition, CNRS UMR 5105, Grenoble, Fr
}

\begin{abstract}
In this paper, we reconsider to what extent, in the context of Neuroscience and Computer Science of the XXIst century, consciousness would remain or not a human specificity. Equivalent functional faculties can be produced by systems differing not only by the material of which they are constructed, but also by the architecture that combines their components. Consequently, is the advent of a consciousness other than ours so inconceivable? What kind of consciousness entities without a cerebral cortex could have?
\end{abstract}

\section{Consciousness as an emerging function of the human brain}

To date in zoological terminology ${ }^{1}$, consciousness has been largely considered as a function specific to Homo sapiens. Beyond this principally Western notion, philosophical approaches initiated in the second half of the 20th century, with anti-speciesism on the one hand (Singer, 1975) and computational singularity on the other (Chalmers, 2010; Good, 1965), share the same idea that some non-human living entities and machines enjoy faculties comparable to those of a conscious human. The moral stakes implied are important. For several centuries, the debate has remained underground among the French scientific community. Directly in line with Descartes's philosophical work, experimental physiology inherited from Claude Bernard (Bernard, 1865), considered the human body as a mechanism similar to that of other animals. The mathematician philosopher, in a dualistic vision separating matter and spirit, distinguished a divine part, the soul, present only in humans. His vision of the soul concorded with religious beliefs of the embodied God, creator of humans in his own image, free and superior to nature and to the entities which compose it. These spiritual notions remained outside the monodisciplinary field of scientists and beyond the reach of their exploratory tools. Scientists thus concentrated their efforts exploring the animal-machine. However, the privileged status of humans at the top of the hierarchy of all living entities, was relativized in 1859 by Darwin's theory of the evolution of species. Neuropsychology, supported more recently by neuroimaging, has directly linked some cortical networks, often common to different species, to specific cognitive and sensitive faculties. Moreover, from a functional point of view, the capacities of present day machines, in terms of memory capacity and information processing abilities, bring them ever closer to living organisms and further widen the gap with the first clocks built centuries ago.

Descartes proposed that, unlike humans, for machines "which had organs and the figure of an ape, or of some animal without reason, we would have no way of recognizing that they would not be in all respects of the same nature as animals" (Descartes, 1637). He thus

\footnotetext{
${ }^{1}$ from Latin homo: human being, and sapiens: discerning, wise, sensible.

2 Perrault considered that an animal is "a being with feeling and which is capable of exercising the functions of life through a soul"; and that this soul "uses the organs of the body, acting as true machines, to coordinate and as the principal cause of actions performed by each part of the machine" (our English translation).
} 
underlined the dichotomy between humans and mere natural or artificial mechanisms. In direct relation to this notion, the French inventor J. de Vaucanson built his famous defecating duck, an automaton reproducing the movements of a feeding duck, which was opposed by other intellectuals, such as Perrault in his "Avertissement" introducing "La mechanique des animaux" (Perrault, 1680) $)^{2}$, and then Diderot and (Le Rond) D'Alembert, 1751-72, who readily granted a soul to animals in their famous Encyclopedia.

Here, associating soul and consciousness, we reconsider to what extent, in the context of Neuroscience and Computer Science of the XXIst century, consciousness would remain or not a human specificity. We then consider what kind of consciousness entities without a cerebral cortex could have.

We start from a materialist, monistic perspective, shared by the majority of neuroscientists. In this perspective, the mind does not exist outside the matter which is its origin. The consciousness evoked here does not refer, like Descartes' soul, to God. Here, consciousness is considered as a functional faculty underpinned by an adequate structure, like other faculties, processes or procedures of our organs (locomotion, communication, reproduction, ...). The question of the attribution of a consciousness to non-human agents fulfilling these functions is thus left largely open. The various forms of the human consciousness emerge from the specific organization of our nervous system, in particular the cerebral cortex. We can examine whether the key neuronal structures and their organization are also present in other animals (see Inset 1). We can also define functional characteristics of consciousness (see Inset 2) and investigate whether they are expressed in other natural or artificial agents. Consciousness can then emerge from substrates that, while non-human, possess equivalent organizational principles that remain for natural agents to be discovered, and for artificial agents to be bio-inspired from humans.

Any emergence of a consciousness conferred to an artificial system would be in line of numerous technological innovations over the last century. Artistic representations of the world (parietal art, painting or sculpture) reside in the use of specific techniques which reproduce some of our capacities for example to perceive a scene and to memorize it. Photography has integrated these biological functions even more effectively: the camera focuses light information through its lenses in a manner similar to the vertebrate eye with its cornea and lens; it has a sensitive matrix (silver film or digital sensor), like its natural model the retina, capable of transcoding light energy into a chemical trace or electrical signal; likewise the photographic image keeps track of a spatial pattern as does the natural memory platform, the cerebral hippocampus. Similarly, the muscular driving force has inspired a man-made functional equivalent in heat engines and then in electric motors. Genetic algorithms, derived from the Darwinian principle of biological evolution, may be used in computer science for solving optimization problems. Finally, the construction of artificial neural networks is bio-inspired (see Inset 3). Just as aeronautics has allowed an artificial production of the function "to fly" without flapping wings, a "human-like" consciousness making use of original devices and mechanisms could be considered without involving a direct imitation of biological neuronal implementation.

Natural history is littered with similar episodes that preceded human "innovations". By evolutionary convergence, very equivalent functions have emerged from organs constructed from distinct tissues at different points throughout the evolutionary tree: the flight of insects and birds, track scent-marking by ants and dogs, and of course the camera-like eye of vertebrates and that of cephalopods such as the octopus. Equivalent functional faculties can thus be produced by systems differing not only by the material of which they are constructed, but also by the architecture that combines their components. Consequently, is the advent of a consciousness other than ours so inconceivable? 
Inset 1: Structural prerequisites. Non-human primates, and even all mammals, have a neocortex with a characteristic six-layered structure. It is the dynamics of flow of signals in this cortex that is at the center of a dominant theory of human consciousness. By comparing the neuronal dynamics of humans declared conscious or unconscious, Tononi identified physiological markers (Tononi, 2004). In such a perspective, an awake mammalian brain can undoubtedly answer with "yes" to the fateful question: "Spirit (or rather, Consciousness), are you there"? Such a notion would thus undermine the distinction between humans and animals proposed by Descartes (Descartes, 1637) based on probability and likelihood considerations: "they [the animals] would have an immortal soul just as we do; which is not probable, because there is no reason to believe it of some animals, without believing it of all, and because there are several, such as oysters, sponges, etc.., too structurally imperfect to be able to believe it of them" (our English translation) ${ }^{3}$.

\section{Functional approach}

According to the functional approach, a first step is to define the properties of a functional consciousness, in order to be able to examine to what extent machines or animals can exhibit it. A proposal is presented in Inset 2. It includes eight criteria of information processing, from perception to introspection. Let us note that this proposal is not only anthropomorphic (inevitably), but more specifically, ontologically and epistemologically, defined by authors whose views are influenced by the use of computerized tools and familiarity with cybernetic concepts. Thus, Seth and Tsakaris summon the "theory of control", of "free energy" and of "predictive coding", to show that the very experience of self can be understood in terms of predictive regulation and introspective inferences. Where Descartes saw in the animal-machine an impossibility in the emergence of the "soul", Seth and Tsakaris turn the problem around considering that it is this very nature of information processing by the "beast machine", that leads to the emergence of our own individuality (Seth and Tsakiris, 2018), in other words a conscious self. Thus, the functional prerequisites we retain for the attribution of consciousness (inset 2) will likely be found in our intelligent machines rather than in our pets.

However, attributes of consciousness (or soul) considered in different cultural environments, reveal distinct concerns and require specific formulations. For example, animal lovers will be more sensitive to dimensions such as the affect, as privileged by Descartes ${ }^{4}$. He proposed six "primitive passions of the soul": admiration, love, hate, desire, joy and sadness (to which one can add the "actions of the soul"). More recently, Birch et al (Birch et al., 2020) proposed five dimensions to evaluate states of consciousness in animals: perceptual richness of the environment (P-richness), which qualifies the precision of discrimination; richness of affective experience (Evaluative-richness), the ability to integrate information at a time (Integration at a time) and across time; and finally, self-consciousness.

\footnotetext{
3 In his letter to Morus on February 5, 1649, Descartes also says that one is more likely to conceive of earthworms, gnats or caterpillars as machines than to attribute an immortal soul to them (AT, V, 277).

${ }^{4}$ Descartes did not make the mistake of neglecting the importance of emotions in human decision making, a mistake that Damasio falsely attributes to him (Damasio, 1992).
} 
Inset 2: Functional prerequisites. Over the last century, cybernetics has helped shed some light on the essential functionalities to consider an agent as being "conscious".

1. Perception and making information available for attentional tasks;

2. Goal management: with priorities - the most essential being survival and reproduction - that guide the choice of actions;

3. Attention focusing to allocate cognitive resources in real time;

4. Appraisal and combination of perceived information which can produce emotions (Scherer, 1999);

5. Action planning and decision-making: based on internal state (especially emotional), goals, analyzed perceptions (including spatial and temporal dimensions), and possible actions and their expected effects;

6. Interaction and communication with the outside world and communicating (in the case of social beings);

7. Learning: to adapt and improve future actions;

8. Introspection: to learn from one's past, to analyze one's own behavior, to understand the behavior of others as a variant of one's own functioning, to decentralize oneself and thus to better understand one's own place in the world (notion of Metacognition).

\section{Other natural consciousnesses}

Considering the above-listed functional prerequisites, animals generally satisfy the first seven. Indeed, high-level cognitive abilities and phenomenal awareness are observable in birds, including parrots and corvids (Gunturkun and Bugnyar, 2016; Nieder et al., 2020). For example, a blue jay seeing "itself" in a mirror attempts to wipe a spot under its bill just as it would from one detected directly on its wing. Moreover, while the structure of the octopus nervous system differs radically from ours (Young, 1971), extensive studies have demonstrated cognitive abilities similar to those of mammals (Schnell et al., 2021). Having bridged the gap separating us from other animals, and then travelled along the path that separates us from non-mammals, would these latter findings in the octopus be essentially crossing the barrier that separates us from invertebrates which lack a central nervous system? Recognition of their intelligence has been made in France by a change in legislation imposing, as for vertebrates, particular precautions in experiments inflicted on cephalopods in genera $1^{5}$. Despite discoveries made by animal behaviorists during the last century, other invertebrates have remained excluded from any consideration as animals having consciousness. From as early as the beginning of the last century Turner described how an ant, isolated on an island in a puddle, organizes the construction of a bridge by assembling objects of different materials (Galpayage Dona and Chittka, 2020). T. Seeley showed that collective decision-making in bees, for example for the selection of the best site for the colony during swarming, emerges from the confrontation of different individual proposals based on which the inhabitants make a choice (Seeley, 2011).

The mathematical model proposed by Tononi (Tononi, 2004), applied to the nervous system of the octopus, would perhaps make it possible to distinguish an awake from an anaesthetized individual. In this model, the criteria retained to recognize a conscious state are based on an evaluation of the entropy (degree of organization) in the configuration of the active connections between the different parts of the human central nervous system. In this aquatic mollusk, the absence of a skull, which maintains the anatomical structures in place, and the lack of hemoglobin, among other things, limit for the moment the use of functional imaging

\footnotetext{
5 Directive 201063/EU of the European Parliament and of the Council of 22 September 2010 on the protection of animals used for scientific purposes.
} 
techniques to highlight the active nervous networks in these two states. Needless to say, that today's techniques being inadequate for assessing the nervous system of an octopus, they are even less able to assess that of an ant!

\section{Artificial consciousnesses}

No one can ignore the cognitive capabilities of present day computers, especially since the rise of machine-learning algorithms which have demonstrated a huge impact on a computer's capacity to excel in complex strategy games, such as Go (Silver et al., 2017). Computers even show intuition to dynamically adapt their strategy based on available incomplete information, typical of the no-limit poker game (Moravčík et al., 2017). On the one hand, these impressively performing algorithms are bio-inspired (see Inset 3), and on the other hand, current neuroscientific theories of human consciousness rely on computational models of information processing. In this hybrid context, the emergence of consciousness in the machine, i.e., the computer, then poses no insurmountable conceptual problem (Dehaene et al., 2017). Indeed, since information processing inspired by the human brain is the seminal principle behind consciousness, computer science is thus the most appropriate technology to serve as a basis for an artificial consciousness.

However, we can clearly see that there is a self-referential mechanism between artificial and natural consciousness, in the manner of Hofstadter's strange loops (Hofstadter, 1979), where a system defines itself only by explicitly referring to itself (Hofstadter's examples are Escher's looped drawings, Bach's sequences or the mathematical logic that leads to the Gödel's incompleteness theorem). Indeed, from the first works on information theory and the birth of computer science, scientists have recognized the connection between the brain and Von Neuman's machine. Machine inventor and father of the current architecture of computers, Von Neuman wrote the book "Brain \& Machine" that was published posthumously (Von Neuman, 1958). Later, N. Wiener, father of cybernetics and source of inspiration for behaviorism, published "Nerve, Brain and Memory models" (Wiener and Shadé, 1963). The founding fathers of Artificial Intelligence J. McCarthy, M. Minsky and A. Newell, were interested in making machines conscious of their mental states (McCarthy, 1995), questioning their free will (McCarthy, 2000), the emergence of the mind via interactions between rational agents (Minsky, 1986) or proposing a computational model of cognition (Newell, 1994). The current main neuroscientific theories of consciousness are derived directly from these two latter works (Newman et al., 1997).

However, the two main theories do not converge on how a machine can be considered as being conscious. On the one hand, the "Global Neuronal Workspace" theory considers consciousness as a kind of global processing of available information in a common space accessible to all mental processes (Baars, 2002; Dehaene et al., 2014; Mashour et al., 2020). There is therefore, in principle, no impediment to a machine being conscious (Dehaene et al., 2017). This theory is directly based on work carried out in artificial intelligence, which considered that cognition emerged from distributed processors (or agents) that wrote on a blackboard visible to all the agents (Minsky, 1986; Newell, 1994). In practice, the design of such machines has incurred some problems of control in favoring access to this global space to certain agents according to the context and allowing final decision-making in real time (HayesRoth, 1982). It should be noted that the decision-making implemented in bees for the management of complex situations has not yet been simulated on a computer. The number of neurons in the bee is estimated at 1 million, compared to 100 billion in humans, and a colony contains about 40,000 individuals. Individual human decision-making and the difficulty in its modeling therefore seems a long way off (Seeley, 2011).

On the other hand, according to the Integrated Information Theory (Tononi, 2004), low values of $\Phi$, a measure of phenomenal awareness, can be produced in systems much simpler than 
the human brain, provided that they contain reciprocally interacting logical units. This condition is certainly fulfilled by the nervous systems of many animals, but also by isolated living cells, and even to a much lesser degree by simple electronic circuits.

Our functional requirements (see Inset 2) are largely met by current machines:

1. Perception: image recognition algorithms (face, person and object detection) and sound recognition algorithms (voice and music recognition) have made great progress, especially since the introduction of deep learning (see Inset 3 ) and the improvement of sensors (LIDAR, high-definition cameras, microphone arrays, etc.);

2. Goal management: in robotics, goals are prioritized by a dynamically assigned score in order to develop a contextual action plan (see point 5);

3. Attention focusing: current robots and machines generally have limited applications that reduces the complexity of resource allocation. However, the heuristics followed for example by a chess program to choose which actions it will perform in the given time (in the absence of an exhaustive analysis), can be considered as a form of attentional selection;

4. Analysis: the modeling of knowledge and reasoning, either inductive or deductive, has been extensively explored in artificial intelligence with the introduction of ontologies and the extension of formal logics (temporal logics, fuzzy logics, ...) and the use of specific languages, based on rules and objects, logic programming (Prolog, LISP languages), by constraints, or query languages for linked data [SPARQL, Semantic Web];

5. Action planning and decision-making: can be seen as an optimization problem. All robots and autonomous systems make decisions (to act or not to act), depending on the context, the expected results of their action(s) and the time available;

6. Interaction and communication with the outside world: an artificial agent in a video game or a robot can exercise the same repertoire of actions as a human being: they can move, grasp objects, turn their head, climb a staircase, emit sounds or express an emotion; they can also communicate via a specific interface (screen, synthetic speech, etc.)

7. Learning: learning has been an essential domain of artificial intelligence since its origin. It is generally based on the observed effect of an action in a given situation in order to adapt future responses in a similar situation. Currently, however, generalization and adaptability capabilities are limited.

8. Introspection: although introspection and self-awareness are essential for a machine to be recognized as having consciousness, both capacities remain underexplored in practice. We can note that current-day machines do perform tasks without action from their user including automatic updating and server interrogation. These functions impose the automatic recognition by the machine of its current state and the necessity to evolve it.

If we take a functionalist point of view on consciousness, we can dismiss certain objections such as the incapacity of a machine to be anything other than an imitation of human consciousness, which is unprovable, in the same way that we cannot prove that any individual, other than oneself, is not a mere mechanical imitation of our consciousness. In this point of view, consciousness has a role and usefulness justified by its emergence and selection during the evolution of biological organisms. Certain criteria, notably the eighth (introspection), once clearly defined, represent a challenge for the designers of intelligent machines.

However, while asking someone to define an object, and then asking a craftsman or engineer to design a prototype with the criteria and attributes mentioned (shapes, colors, 
physical properties...) is easy, the result will undoubtedly be unsatisfactory. Indeed, even if rationally the prototype perfectly satisfies the definition according to the first specifications to the engineer, new criteria will be imposed. For a computer program, the criticism of the result can be taken into account repeatedly for a beta version, then beta..+ The expression "artificial intelligence" has long been ridiculed with sceptics arguing the incapacity of computers to play chess, to identify faces, to recognize a language or a text, to translate it (Searle, 1983), or to imagine new forms etc. and used to put our minds at rest about the superiority of human intelligence, which had long been unequaled. According to Chapouthier and Kaplan (Chapouthier and Kaplan, 2011), the race towards proving artificial intelligence is endless: as soon as a criterion showing a machine's failure to match human ability is solved, after a "yes but...", a new challenge can be launched. After years of challenging machines using as a paradigm the board game Go, which requires a form of intuition beyond formal reasoning, machines have prevailed against the greatest master (see the AlphaGo vs Lee Sedol match in 2016, (Silver et al., 20)). Clearly today, artificial intelligence has become widely recognized as a form of intelligence, partly equivalent, partly even superior to that of human individuals. At what point we will be able to accept the existence of an artificial consciousness, even different from human consciousness remains an open debate. Some will maintain that any artificial consciousness will always lack free will.

However, any sufficiently complex artificial consciousness, influenced by many factors (its capabilities, its objectives, its emotions, its memory, etc.) and evolving continuously by learning, will have a complex behavior, sometimes predictable, sometimes unexpected, while remaining perfectly deterministic if we know all the factors involved. In the same way, social psychology, sociology and anthropology show that our behavior is influenced by social norms, the socio-economic environment in which we live and our life experiences. Since we cannot know all the factors influencing an individual (genetic, epigenetic, unconscious mechanisms, dietary, life course, emotional state, motivations, personal and social norms, ability to project oneself into the future, etc.), it is difficult to predict one's behavior, although statistical data do exist on the correlation between these factors and individual behavior. We can therefore defend the idea that free will, often associated exclusively with human consciousness, is in reality an illusion that hides a deterministic behavior the influencing factors and unconscious mechanisms of which escape us (Bignetti, 2014; Soon et al., 2008). Being free to make decisions requires that they be made by oneself and adapted to a dynamic situation. What we learn from the study of insects is that this does not necessarily imply awareness (Heisenberg, 2009), unless we grant consciousness to a group of insects. Altogether, these findings have helped build a solid framework on which to base arguments favoring "free will even for robots" (McCarthy, 2000).

In conclusion, the question we should be asking ourselves is probably not whether a machine is capable of consciousness or not ("any more than whether a submarine can swim" E. Dijkstra) but rather whether the machine represents a good model to help us understand the conditions of emergence of states of consciousness. In other words, we should be exploring which kinds of systems are conscious and which are not, and why; and how these states of consciousness help or hinder the resolution of certain complex tasks and participate in the definition of the individuality of living entities. Increased efforts to understand consciousness and the study of computational architectures implementing models of sensory-motor and cognitive processing (Schrimpf et al., 2018), testable on shared datasets, are key to refining our current theories of Homo Sapiens uniqueness. 


\section{Inset 3: Neural networks}

The principle dates back to the last century during the sixties, and comes from the connectionist branch of the new discipline at that time, artificial intelligence. The idea is to mimic brain function by grouping together formal neurons. A formal neuron is a simple entity whose output depends on a function (step, sigmoid...) and on the weight attributed to its inputs. A neural network consists of successive layers whose outputs are connected. During a phase called "supervised learning", examples (e.g. images of insects and arachnids) are presented at the input of the system. An error function is estimated to compare the obtained output (e.g. insect vs arachnid) with the expected one (e.g. arachnid). The weights associated to each layer of neurons are then adapted to reduce the error function. A phase called "Validation" allows the refining of weights on a few examples if necessary. Finally, the "test" phase evaluates the performance of the network on a new set of examples. A single-layer system, such as the perceptron (Rosenblatt, 1958), allows the linear separation of two classes. In practice, image classification is not a linear operation and several layers (we speak of hidden layers, hence the name deeplearning, for the layers located between the input and the output) are therefore necessary. As soon as they were introduced, multi-layer networks proved to be difficult to program and this line of research was quickly abandoned (Minsky and Papert, 1969). It came back in force at the beginning of the 21 st century thanks to the conjunction of three major factors: the increased power of computers, the availability of large amounts of data based on which a model could be assessed as being generalizable or not, and the development of an efficient method of adjusting the weights during training (the so-called back-propagation method, (Rumelhart et al., 1986)). Note that instead of associating each artificial neuron to one image pixel, which is very computationally expensive, Y. Le Cun et al. proposed the association to local information obtained within a neighborhood (Le Cun et al., 1989). They used convolutional filters (CNN: Convolutional Neural Network) that vary at each layer. This was originally a bio-inspired approach to the mammalian visual system, based on the seminal work of Fukushima on the NeoCognitron (Fukushima, 1980).

\section{Contributions}

The three authors collectively wrote this chapter; the other members of the CARMEN collective reviewed its contents, suggested some changes and made insightful comments.

\section{References}

Baars, B., 2002. The conscious access hypothesis: origins and recent evidence. Trends Cognitive Sciences 6, 47-52.

Bernard, C., 1865. Introduction à l'étude la médecine expérimentale. Flammarion, Paris, Fr (ed. 1984).

Bignetti, E., 2014. The functional role of free-will illusion in cognition: "The Bignetti Model". Cognitive Systems Research 31-32, 45-60.

Birch, J., Schnell, A.K., Clayton, N.S., 2020. Dimensions of Animal Consciousness. Trends Cogn Sci 24, 789-801.

Chalmers, D.J., 2010. The Singularity: A Philosophical Analysis. Journal of Consciousness Studies

17, 7-65.

Chapouthier, G., Kaplan, F., 2011. L'homme, l'animal et la machine : perpétuelles redéfinitions

CNRS editions Paris, Fr.

Darwin, C., 1859. L'origine des espèces. Flammarion Paris, Fr (1992). 
Dehaene, S., Charles, L., King, J.R., Marti, S., 2014. Toward a computational theory of conscious processing. Curr Opin Neurobiol 25, 76-84.

Dehaene, S., Lau, H., Kouider, S., 2017. What is consciousness, and could machines have it? Science 358, 486-492.

Descartes, R., 1637. Discours de la méthode. Vrin Paris, Fr (1984).

Diderot, D., (Le Rond) D’Alembert, J., 1751-72. L'Encyclopédie ou Dictionnaire raisonné des sciences, des arts et des métiers.

Fukushima, K., 1980. A self-organizing neural network model for a mechanism of pattern recognition unaffected by shift in position. Biological Cybernetics 36, 193-202.

Galpayage Dona, H., -Chittka, L., 2020. Charles H. Turner, pioneer in animal cognition. Science 370, 530-531.

Good, I.J., 1965. Speculations Concerning the First Ultraintelligent Machine. In: Alt, F.L., Rubinoff, M. (Eds.), Advances in Computers. Academic Press

, New York City, pp. 31-88.

Gunturkun, O., Bugnyar, T., 2016. Cognition without Cortex. Trends Cogn Sci 20, 291-303.

Hayes-Roth, B., 1982. A blackboard architecture for control. Artificial Intellligence 26, 251321.

Heisenberg, M., 2009. Is free will an illusion. Nature 459, 164-165.

Hofstadter, D., 1979. Gödel, Escher, Bach: an Eternal Golden Braid. Basic Books.

Le Cun, Y., Boser, B., Denker, J., Henderson, D., Howard, R., Hubbard, W., Jackel, L., 1989.

Handwritten Digit Recognition with a Back-Propagation Network NIPS, pp. 396-404.

Mashour, G.A., Roelfsema, P., Changeux, J.P., Dehaene, S., 2020. Conscious Processing and the Global Neuronal Workspace Hypothesis. Neuron 105, 776-798.

McCarthy, J., 1995. Making robots conscious of their mental states. MachineIntelligence 15, Oxford Univ.

McCarthy, J., 2000. Free will - even for robots. Stanford Univ.

Michel, M., Beck, D., Block, N., Blumenfeld, H., Brown, R., Carmel, D., Carrasco, M., Chirimuuta, M., Chun, M., Cleeremans, A., Dehaene, S., Fleming, S.M., Frith, C., Haggard, P., He, B.J., Heyes, C., Goodale, M.A., Irvine, L., Kawato, M., Kentridge, R., King, J.R., Knight, R.T., Kouider, S., Lamme, V., Lamy, D., Lau, H., Laureys, S., LeDoux, J., Lin, Y.T., Liu, K., Macknik, S.L., Martinez-Conde, S., Mashour, G.A., Melloni, L., Miracchi, L., Mylopoulos, M., Naccache, L., Owen, A.M., Passingham, R.E., Pessoa, L., Peters, M.A.K., Rahnev, D., Ro, T., Rosenthal, D., Sasaki, Y., Sergent, C., Solovey, G., Schiff, N.D., Seth, A., Tallon-Baudry, C., Tamietto, M., Tong, F., van Gaal, S., Vlassova, A., Watanabe, T., Weisberg, J., Yan, K., Yoshida, M., 2019. Opportunities and challenges for a maturing science of consciousness. Nat Hum Behav 3, 104-107.

Minsky, M., 1986. The Society of Mind. Simon \& Schuster, New York.

Minsky, M., Papert, S., 1969. Perceptrons: An Introduction to Computational Geometry. The MIT Press, Cambridge, MA.

Moravčík, M., Schmid, M., Burch, N., Lisý, V., Morrill, D., Bard, N., Davis, T., Waugh, K., Johanson, M., Bowling, M., 2017. DeepStack: Expert-level artificial intelligence in heads-up no-limit poker. Science 356, 508-513.

Newell, A., 1994. Unified theory of cognition. Harvard University Press.

Newman, J., Baars, B., Cho, S.-B., 1997. A Neural Global Workspace Model for Conscious Attention. Neural Netw 10, 1195-1205.

Nieder, A., Wagener, L., Rinnert, P., 2020. <Corvidé.pdf>. Science 369, 1626-1629.

Perrault, C., 1680. La Méchanique des animaux. Coignard, Paris (Fr).

Rosenblatt, F., 1958. The perceptron: a probabilistic model for information storage and organization in the brain. Psychol Rev 65, 386-408. 
Rumelhart, D.E., Hinton, G.E., Williams, R.J., 1986. Learning representations by backpropagating errors. Nature 323, 533-536.

Scherer, K.R., 1999. Appraisal theory. Handbook of cognition and emotion. John Wiley \& Sons, pp. 637-663.

Schnell, A.K., Amodio, P., Boeckle, M., Clayton, N.S., 2021. How intelligent is a cephalopod? Lessons from comparative cognition. Biol Rev Camb Philos Soc 96, 162-178.

Schrimpf, M., Kubilius, J., Hong, H., Majaj, N.J., Rajalingham, R., Issa, E.B., Kar, K., Bashivan, P., Prescott-Roy, J., Schmidt, K., Yamins, D.L.K., DiCarlo, J.J., 2018.

Searle, J., 1983. Minds, brains and programs. In: Hofstadter, D.R., Dennett, D.C. (Eds.), The Mind's I: fantasies and reflections on self and soul. Penguin Books, Hardmonsworth, pp. 353373.

Seeley, T.D., 2011. Honeybee democracy. Princeton University Press.

Seth, A.K., Tsakiris, M., 2018. Being a Beast Machine: The Somatic Basis of Selfhood. Trends Cogn Sci 22, 969-981.

Silver, D., Schrittwieser, J., Simonyan, K., Antonoglou, I., Huang, A., Guez, A., Hubert, T., Baker, L., Lai, M., Bolton, A., Chen, Y., Lillicrap, T., Hui, F., Sifre, L., van den Driessche, G., Graepel, T., Hassabis, D., 2017. Mastering the game of Go without human knowledge. Nature 550, 354-359.

Singer, P., 1975. Animal Liberation. HarperCollins, NY, USA.

Soon, C.S., Brass, M., Heinze, H.J., Haynes, J.D., 2008. Unconscious determinants of free decisions in the human brain. Nat Neurosci 11, 543-545.

Tononi, G., 2004. An information integration theory of consciousness. BMC Neurosci 5, 42.

Von Neuman, J., 1958. The computer and the Brain. Yale University Press, New Haven. Wiener, N., Shadé, J., 1963. Nerve, Brain and Memory Models. Elsevier, New York.

Young, J.Z., 1971. Anatomy of the nervous system of Octopus vulgaris. . Clarendon Press, Oxford (UK). 